\title{
Outcomes of Late Open Conversion after Endovascular Abdominal Aneurysm Repair
}

\author{
Yoshikatsu Nomura, MD, PhD, ${ }^{1}$ Kanetsugu Nagao, MD, ${ }^{1}$ Shota Hasegawa, MD, ${ }^{1}$ \\ Motoharu Kawashima, MD, ${ }^{1}$ Takanori Tsujimoto, MD, ${ }^{1}$ So Izumi, MD, PhD, ${ }^{1}$ \\ Masamichi Matsumori, MD, PhD, ${ }^{1}$ Hiroshi Tanaka, MD, PhD, ${ }^{1}$ Hirohisa Murakami, MD, PhD, ${ }^{1}$ \\ Tasuku Honda, MD, PhD, ${ }^{1}$ Ryota Kawasaki, MD, PhD, ${ }^{2}$ and Nobuhiko Mukohara, MD, PhD ${ }^{1}$
}

\begin{abstract}
Objective: To review our experience with a late open conversion as a final option for an endograft infection and aneurysm expansion after endovascular aneurysm repair (EVAR), especially in endoleaks for which radiological intervention is impossible.
\end{abstract}

Materials and Methods: In this retrospective study, 13 late open conversions out of 513 consecutive patients treated by EVAR were analyzed. Indications for an open conversion were aneurysm enlargement, including all endoleaks, endograft migration, and endograft infection. The patients' data on demographics, operative details, and outcomes were reviewed.

Results: Indications for a late open conversion included endoleaks, infection, and migration in $61.5 \%, 30.8 \%$, and $7.7 \%$ of patients, respectively. The median interval from the initial EVAR was 32.4 months. Complete endograft explantation was performed in four patients with an endograft infection. In endoleak cases, the endograft was partially preserved and a neo-neck was used. Sacotomy and branch ligation were performed in one case. One major operative complication was an aortic injury during infrarenal aortic cross-clamping in an endograft migration case. There was no operative mortality.

Conclusion: A late open conversion after EVAR is valuable as a final option. The aortic cross-clamp site, especially in endograft migration cases, should be carefully considered.

${ }^{1}$ Department of Cardiovascular Surgery, Hyogo Brain and Heart Center at Himeji, Himeji, Hyogo, Japan

${ }^{2}$ Department of Radiology, Hyogo Brain and Heart Center at Himeji, Himeji, Hyogo, Japan

Received: January 14, 2019; Accepted: March 22, 2019 Corresponding author: Yoshikatsu Nomura, MD, PhD. Department of Cardiovascular Surgery, Hyogo Brain and Heart Center at Himeji, 520 Saisho-ko, Himeji, Hyogo 670-0981, Japan

Tel: +81-79-293-3131, Fax: +81-79-295-8199

E-mail: y_katsu1027@yahoo.co.jp

(cc) BY-NC-SA (92019 The Editorial Committee of Annals of Vascular Diseases. This article is distributed under the terms of the Creative Commons Attribution License, which permits use, distribution, and reproduction in any medium, provided the credit of the original work, a link to the license, and indication of any change are properly given, and the original work is not used for commercial purposes. Remixed or transformed contributions must be distributed under the same license as the original.
To avoid aneurysm-related events, graft replacement is recommended, if possible.

Keywords: late open conversion, aneurysm enlargement, endograft infection

\section{Introduction}

Endovascular aneurysm repair (EVAR) is a widespread and established treatment for an abdominal aortic aneurysm (AAA) because it is less invasive than an open repair (OR). However, EVAR can lead to complications and has a high rate of secondary reinterventions of $\left.9 \%-15 \%{ }^{1-3}\right)$ The majority of reinterventions are caused by endoleaks. Other causes for reintervention include endograft migration, leg occlusion, and endograft infection.

The majority of endoleaks requiring reintervention in the late phase is the type II endoleak. Most secondary interventions for type II endoleaks are successfully performed with a percutaneous catheter intervention, but a small number of patients may require an open conversion (OC) with or without endograft explantation caused by a failed catheter intervention. ${ }^{4-6)}$ The $\mathrm{OC}$ is the last option for catheter reintervention failure and infectious cases.

This study aimed to report the incidence, technical aspects, and outcomes of a single-center experience with $\mathrm{OC}$ after a failed EVAR.

\section{Materials and Methods}

\section{Patient selection}

Patients receiving OC after EVAR between October 2007 and September 2018 were included in this retrospective study. An OC was defined as total or partial endograft removal with prosthetic graft reconstruction or sacotomy and side branch ligation under a transperitoneal or retroperitoneal approach. The indications for an OC were enlargement of the aneurysm diameter due to any type of endoleak that is untreatable by catheter intervention, endograft infection, and endograft migration. The cases 
with a thrombectomy for endograft limb thrombosis were excluded in this study.

OC was chosen as the treatment strategy for type I or III endoleaks that could not be treated by endovascular techniques because of inadequate anatomy. For type II endoleaks, if the aneurysm diameter increased by $5 \mathrm{~mm}$ from the initial size, a secondary endovascular intervention was performed. The reason for choosing an OC for type II endoleaks was that the endoleak flow could not be controlled with endovascular intervention, and the aneurysm tended to expand.

The type of endograft, duration of implantation of the endograft, reason for an OC, operative details, operative and long-term mortality, and the length of stay were identified. Operative details included the surgical approach, type and site of aortic clamp, type of reconstruction, and complications. A preoperative diagnosis was made using computed tomography (CT) and magnetic resonance imaging (MRI).

This study was approved by the institutional review board at our center.

\section{Statistical analysis}

A statistical analysis was conducted using JMP $^{\circledR}$ (SAS Institute Inc., Cary, NC, USA). Continuous variables were presented as the mean \pm standard deviation or the median and categorical variables as percentages. An ANOVA was used for comparison between multiple groups.

\section{Results}

During this period, a total of 513 EVARs were performed. Secondary interventions including an OC were performed in 49 cases $(9.5 \%)$. Secondary catheter embolization for a type II endoleak was performed 50 times in 31 cases. During the same period, 13 patients $(2.5 \%)$ required a late OC. Twelve patients were male $(92.3 \%)$, and the mean age was $76.1 \pm 4.1$ years (range: $71-82$ years). The indications for a late OC were eight patients $(61.5 \%)$ with endoleaks, four patients $(30.8 \%)$ with an endograft infection, and one patient $(7.7 \%)$ with endograft migration. There was no rupture case in this study. $\mathrm{Pa}-$ tient characteristics, indications, and comorbidities are presented in Table 1. All cases were treated within each device of instructions for use. The OC rate of each device was Zenith (Cook Medical, Bloomingtom, IN, USA) in $6.1 \%$, Excluder (W. L. Gore \& Ass., Flagstaff, AZ, USA) in $2.0 \%$, and Endurant (Medtronic, Santa Rosa, CA, USA) in $2.6 \%$. There was no significant difference between the device and the OC $(p=0.29)$. The median time from the initial EVAR to an OC was 32.4 months (range: 3.9-110.7 months). The mean aneurysm diameter prior to the EVAR was $51.7 \pm 5.2 \mathrm{~mm}$. The decrease of the
Table 1 Patient demographics and comorbidities

\begin{tabular}{lcc}
\hline \multicolumn{1}{c}{ Variable } & No. or mean \pm SD & \% or range \\
\hline Patients, total & 13 & \\
Sex & 12 & \\
$\quad$ Male & $76.1 \pm 4.1$ & $71-82$ \\
Age at OC (years) & & \\
Comorbidities & 10 & 76.9 \\
$\quad$ Hypertension & 5 & 38.5 \\
Renal insufficiency & 2 & 15.4 \\
Coronary artery disease & 2 & 15.4 \\
Cerebrovascular disease & 1 & 7.7 \\
$\quad$ CoPD & & \\
Indication of OC & 8 & 61.5 \\
Endoleak & 4 & 30.8 \\
Infection & 1 & 7.7 \\
Migration & &
\end{tabular}

SD: standard deviation; OC: open conversion; COPD: chronic obstructive pulmonary disease

aneurysm diameter, which was defined as $5 \mathrm{~mm}$ of shrinkage, was recognized in seven cases during the follow-up, but the aneurysm diameter was expanded by endoleaks. The mean aneurysm sac enlargement from the minimum diameter at the follow-up to an OC was $7.5 \pm 5.5 \mathrm{~mm}$, and it was $9.9 \pm 4.6 \mathrm{~mm}$ in endoleak cases. Before the OC, seven patients received 13 endovascular procedures. All endovascular procedures were percutaneous trans-arterial culprit branch embolization.

The indications and aneurysm diameter changes are shown in Table 2. Regarding endoleaks, a preoperative diagnosis by CT or MRI may be different from intraoperative findings. The type IIIb endoleak, which is a leak from the device suture hole, was diagnosed in three cases by an intraoperative examination (Fig. 1). The correct diagnosis rate of preoperative image examination was $62.5 \%$, and type IIIb endoleaks could not be diagnosed using preoperative images. All three cases of type IIIb endoleaks were diagnosed as type II or V endoleaks preoperatively.

There were four cases of endograft infection. The details of the infection were an aorto-enteric fistula (AEF) in two cases, an iatrogenic infection after catheter embolization for a type II endoleak in one case, and unknown in one case.

\section{Operative details}

The operative details are shown in Table 3. All patients were operated upon using the transperitoneal approach via a midline laparotomy. The proximal aortic crossclamp was infrarenal in eight cases $(61.5 \%)$ and suprarenal in four cases $(30.8 \%)$. One suprarenal clamp case required the use of an intra-aortic balloon occlusion (IABO) catheter in the infection group. The distal clamp site was the common iliac artery, including the endograft 
Table 2 Indications for an open conversion and aneurysm diameter changes

\begin{tabular}{|c|c|c|c|c|c|c|c|}
\hline Patient & Indication of conversion & Device & $\begin{array}{c}\text { Number of } \\
\text { secondary } \\
\text { interventions }\end{array}$ & $\begin{array}{l}\text { Initial AAA } \\
\text { diameter } \\
(\mathrm{mm})\end{array}$ & $\begin{array}{l}\text { Minimum } \\
\text { diameter at the } \\
\text { follow-up }(\mathrm{mm})\end{array}$ & $\begin{array}{c}\text { Diameter } \\
\text { at OC } \\
(\mathrm{mm})\end{array}$ & $\begin{array}{l}\text { Time to } \\
\text { conversion } \\
\text { (months) }\end{array}$ \\
\hline 1 & Migration & Endurant & 0 & 50 & 40 & 48 & 30.9 \\
\hline 2 & Type II endoleak & Zenith & 0 & 51 & 51 & 57 & 89.9 \\
\hline 3 & Type II endoleak & Excluder & 1 & 55 & 47 & 63 & 89.0 \\
\hline 4 & Type II endoleak & Excluder & 2 & 48 & 46 & 54 & 28.4 \\
\hline 5 & Type II endoleak & Endurant & 4 & 64 & 64 & 69 & 13.2 \\
\hline 6 & Type II endoleak & Zenith & 2 & 50 & 43 & 55 & 107.3 \\
\hline 7 & Type IIlb endoleak & Zenith & 2 & 46 & 37 & 55 & 110.7 \\
\hline 8 & Type IIIb endoleak & Endurant & 0 & 58 & 58 & 66 & 32.3 \\
\hline 9 & Type IIIb endoleak & Endurant & 1 & 51 & 51 & 61 & 43.5 \\
\hline 10 & Infection, AEF & Excluder & 0 & 56 & 43 & 43 & 67.3 \\
\hline 11 & Infection, AEF & Excluder & 0 & 47 & 47 & 50 & 3.9 \\
\hline 12 & Infection & Excluder & 1 & 48 & 41 & 46 & 22.0 \\
\hline 13 & Infection & Endurant & 0 & 48 & 48 & 48 & 8.7 \\
\hline Mean \pm SD & & & $1.0 \pm 1.2$ & $51.7 \pm 5.2$ & $47.2 \pm 7.4$ & $55.0 \pm 8.0$ & $49.8 \pm 38.2$ \\
\hline
\end{tabular}

SD: standard deviation; AEF: aorto-enteric fistula; AAA: abdominal aortic aneurysm; OC: open conversion

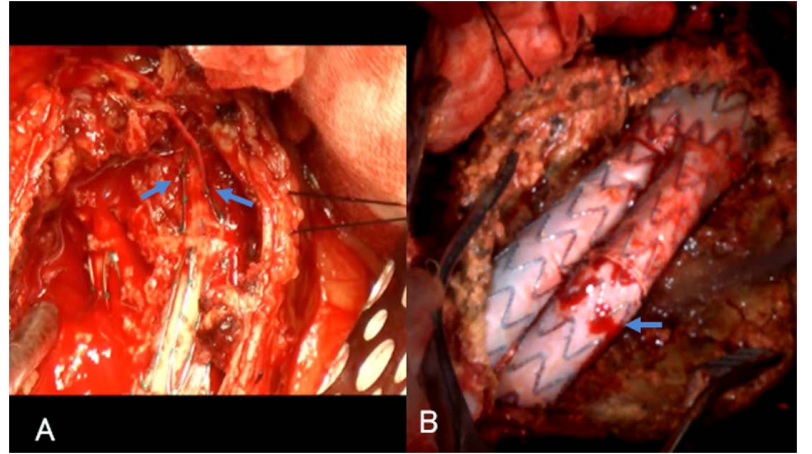

Fig. 1 A type IIlb endoleak from intraoperative findings, showing active bleeding from the suture hole in Zenith (arrows) (A) and oozing from the suture hole in Endurant (arrow) (B).

leg or native external and internal iliac arteries. In endoleak cases, except for a type I endoleak, the aneurysm sac was incised before aortic cross-clamping to detect the location of the leak. After incision of the aneurysmal sac, complete removal of the endograft was performed in three cases. These included all cases of the endograft infection. In only one case of infection, the suprarenal stent was not removed. This case used the IABO catheter. Partial removal of the endograft was performed in the remaining eight cases. After incision of the aneurysmal sac and aortic cross-clamping, the endograft was cut transversely at the level of the main body fabric, and the proximal anastomosis involving the proximally preserved main body, aortic wall, and graft were sutured together ${ }^{7)}$ (Fig. 2). In only one case, the lumbar artery that caused endoleaks was ligated from within the aneurysm.

Prosthetic aortic reconstruction, using a standard $\mathrm{Da}$ cron graft in 11 cases and an expanded polytetrafluoro- ethylene graft in one infection case, was an aorto-bi-iliac bypass in 12 cases. In case of infection, debridement of the infected aneurysm wall and omentopexy were performed. In AEF cases, the duodenal fistula was closed directly.

\section{Early postoperative outcomes}

A major operative complication was aortic injury in one migration case. During aortic cross-clamping, the migrated suprarenal stent stabbed and injured the aorta. Immediately, a suprarenal aortic clamp was inserted, and the aortic injury site was repaired. In this case, renal insufficiency worsened due to renal ischemia, and dialysis was necessary. In other cases, there were no complications, such as an aortic injury even under infrarenal aortic cross-clamp. In case of infection, there was no positive blood culture. Intraoperative cultures from the aortic wall identified Streptococcus anginosus, Pseudomonas aeruginosa, and Enterococcus faecalis in three AEF cases, Staphylococcus epidermidis in an iatrogenic case, and methicillin resistant coagulase-negative staphylococci in an unknown case. Antibiotic therapy was performed in all cases intravenously for 6 weeks after operation. Only one patient was administrated oral antibiotics for the lifelong because this case was in a septic state preoperatively. There was no in-hospital death. The overall incidence of severe complication was $7.7 \%$. The overall mean hospital stay was $29.6 \pm 22.4$ days.

\section{Late outcomes}

The median postoperative follow-up period was 27.9 months (range 2.2-52.8 months). There were two late deaths. One patient developed an acute aortic dissection rupture 3 months after the OC for endograft migration. In 
Table 3 Operative details and early outcomes

\begin{tabular}{|c|c|c|c|c|}
\hline Patient & Operation & Clamp site & Morbidity & Death \\
\hline 1 & Partial EG preservation+bifurcated grafting & Infrarenal $\rightarrow$ suprarenal & Aorta injury, AKI & No \\
\hline 2 & Sacotomy+lumbar artery ligation & None & No & No \\
\hline 3 & Partial EG preservation + bifurcated grafting & Infrarenal & No & No \\
\hline 4 & Partial EG preservation+bifurcated grafting & Infrarenal & No & No \\
\hline 5 & Partial EG preservation+bifurcated grafting & Infrarenal & No & No \\
\hline 6 & Partial EG preservation+bifurcated grafting & Infrarenal & No & No \\
\hline 7 & Partial EG preservation+bifurcated grafting & Infrarenal & No & No \\
\hline 8 & Partial EG preservation+bifurcated grafting & Infrarenal & No & No \\
\hline 9 & Partial EG preservation+bifurcated grafting & Infrarenal & No & No \\
\hline 10 & Complete removal of EG+bifurcated grafting+omentopexy & Suprarenal & No & No \\
\hline 11 & Complete removal of EG+bifurcated grafting+omentopexy & Suprarenal & No & No \\
\hline 12 & Complete removal of EG+bifurcated grafting+omentopexy & Suprarenal & No & No \\
\hline 13 & $\begin{array}{l}\text { Removal of EG+ preserved suprarenal stent+bifurcated } \\
\text { grafting + omentopexy }\end{array}$ & Suprarenal $(\mathrm{IABO}) \rightarrow$ infrarenal & No & No \\
\hline
\end{tabular}

EG: endograft; AKI: acute kidney injury; IABO: intra-aortic balloon occlusion

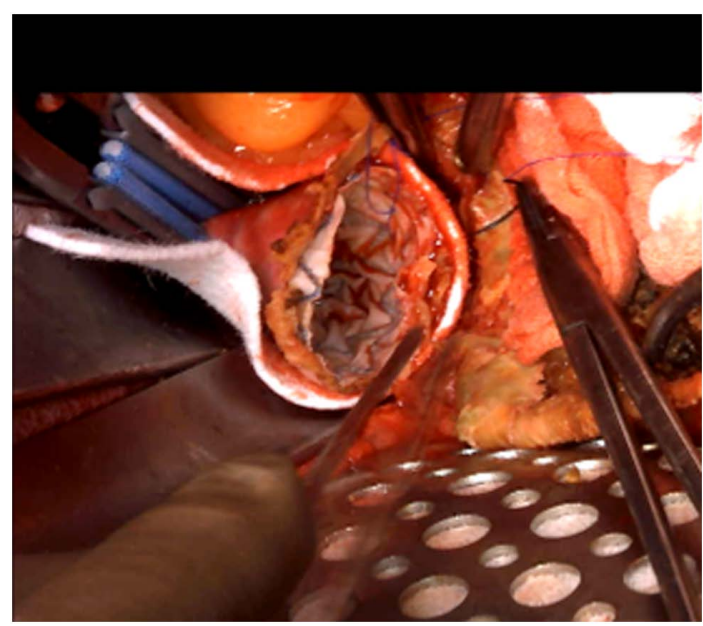

Fig. 2 A partial endograft preservation.

The aortic cross clamp site was on the infrarenal abdominal aorta, including the endograft main body. The proximal anastomosis was sutured together with the proximally preserved main body, aortic wall, and the new graft.

this migration case, an aortic dissection was not detected after the OC, but an aortic injury may have been the cause of the aortic dissection. Another patient developed a purulent spondylitis and sepsis 6 months after the OC for AEF and died. In this infection case, AEF was suspected at the time of the initial EVAR, but there was no sign of infection after the EVAR. A fistula was confirmed at 4 months after the EVAR, and he developed sepsis. OC was performed in this situation, but the infection was already uncontrollable. This patient died of sepsis and purulent spondylitis 6 months after the OC.

The aneurysm sac was enlarged and a new type II endoleak was recognized in the only lumbar artery ligation case. The diameter of the AAA was expanded from $45 \mathrm{~mm}$ to $73 \mathrm{~mm}$ in 2 years after the $\mathrm{OC}$, in this case. There were no aortic-related complications in other cases.

\section{Discussion}

EVAR can be an alternative option for the OR for an AAA because of its excellent early results and low invasiveness, but aneurysm-related mortality has been found to increase on long-term follow-up. ${ }^{8,9)}$ Endoleaks are the disadvantages of the EVAR. The rate of secondary intervention was almost $15 \%$, which is higher than a conventional OR. ${ }^{2,3,8,10)}$ Many reported cases were defined as an enlargement of the AAA diameter above $5 \mathrm{~mm}$ and were indicated as secondary reintervention. ${ }^{11)}$ Late OC was considered a final option when the secondary endovascular procedure failed. There was no clear indication of the aneurysm size in the OC, especially type II endoleaks, but a diameter $>65 \mathrm{~mm}$ was a risk factor for new onset type I endoleaks. ${ }^{11)}$ The initial experience with a late OC reported that the procedure-related mortality rate was as high as $17 \%$, and it was considered a hazardous procedure. ${ }^{12)} \mathrm{A}$ recent literature review showed that the 30-day mortality rate was $9.1 \%$. Mortality rates were different between elective and urgent operation (3.2\% vs. $29.2 \%)$. The incidence of a late OC after failed EVAR was 3.7\%. Endoleak was the most common cause of the OC, followed by infection. ${ }^{13)}$

The removal of the endograft and aortic clamp site are important problems during OC. No clear recommendations exist regarding management of an endograft by a complete or partial removal, and this issue is controversial. Complete removal is an absolute need in infection cases. Complete removal, especially an endograft with suprarenal fixation, may increase the risk of an aortic wall injury. ${ }^{14)}$ Suprarenal fixation by a burb may cause inflammation around the suprarenal aorta, and it may be 
difficult for dissection. Partial removal of the endograft may lead to a lower risk of operative complications in the absence of proximal endoleaks. ${ }^{15)}$ Another problem was that leaving endografts caused delayed complications, and therefore, these cases should be monitored continuously. In our series, we performed infrarenal aortic cross-clamping including the main body and proximal anastomosis using the neo-neck technique. ${ }^{7)}$ The neo-neck technique includes suturing of the aortic wall, remnant endograft body, and new prothesis together. Despite the possibility of a protruding cut-end of the stent, this technique is an effective and safe technique, and complications are not observed in this proximal anastomosis site during the follow-up period. Similarly, Lipsitz et al. reported that there were no complications in the anastomotic site during the 22-month follow-up from the OC. ${ }^{16)}$

Only one patient suffered an aortic injury under the infrarenal cross-clamp in a migration case. At the time of aortic cross-clamping, attention must be paid to the position between the suprarenal stent and aorta. In other infrarenal clamp cases, there was no aortic injury. Dissecting around the aorta was like the conventional OR and is an effective method.

Another treatment option for a persistent type II endoleak was sacotomy and ligation of the culprit arteries. This technique is less invasive than the endograft removal and has the advantage of avoiding aortic cross-clamping. ${ }^{17-19)}$ Although this method is effective for elderly and patients who are high-risk requiring surgery, it has two problems. The first is the endograft dislocation when performing maneuvers for the culprit artery ligation, ${ }^{19)}$ and the second is the recurrence of the endoleak. Moulakakis et al. reported that type Ia and Ib endoleaks occurred because of main body and limb dislocations during ligation of the culprit vessels. ${ }^{19)}$ The recurrence of the endoleak has not been reported, ${ }^{18,20)}$ but ligating all side branches as much as possible is necessary. Additionally, continuously monitoring new onset endoleaks and infections is necessary. In our experience, a new type II endoleak recurred in the follow-up period because all side branches could not be ligated, resulting in expansion of the aneurysm. Therefore, sacotomy and ligation were not recommended as the first-choice treatment, and if it was possible regarding the patient's risk, we decided to remove the endograft, to make this operation the final treatment.

Stent-graft infection (SGI) is reported to occur in $0.2 \%-$ $0.7 \%$ of cases after EVAR. ${ }^{21,22)}$ The etiology of a SGI has not been understood clearly. Bacterial contamination at the time of initial EVAR or secondary intervention, hematogenous infection from another site, and development of AEF may lead to a SGI. ${ }^{22,23)}$ The incidence of a SGI differs depending on the interventional location, and the rate of infection in the interventional radiology room is higher than that in the operating room. ${ }^{24)}$ In this study, one case developed SGI after transcatheter embolization for a type II endoleak. We considered that bacterial contamination occurred when performing the procedure in the interventional radiology room. Since then, we have strictly administered prophylactic antibiotics and have not confirmed the occurrence of procedure-related infection. The diagnosis of SGI is based on clinical symptoms, radiological findings of infection, and microbial cultures, according to a Management of Aortic Infection Collaboration consensus. ${ }^{25)} \mathrm{A}$ recent systematic review of the SGI showed that in-hospital mortality was very high, at $26.6 \%$ with an OC and $63.3 \%$ with conservative treatment. ${ }^{26)}$ The cases with an enteric fistula had a poorer prognosis than those without a fistula. ${ }^{27)}$ Regarding treatment, if the patients' general conditions allow, an OR for complete removal of the infected endograft is preferable. Although this is common in both infection and endoleak cases, there are reports on the complete removal of endografts using the ice slush method, ${ }^{28)}$ the Rumel tourniquet method, ${ }^{23)}$ and using a 20 -ml syringe techique. ${ }^{23,29)}$ Regarding the reconstruction, an extra-anatomical bypass has the high risks of infectionrelated complications such as aortic stump blowout and graft occlusion, compared to in-situ reconstruction. Youn et al. suggested that the extra-anatomical bypass should be performed in cases with gross pus or remnant infection, whereas in-situ reconstruction is recommended for cases with good antibiotic reactivity. ${ }^{30)}$ If the omentum cannot be used, autogenous vein transplantation using a common femoral vein should be considered. In this study, in-situ reconstruction using a vascular prosthesis and omentopexy was performed because the local infection was controllable. The recurrence of local infection was not observed postoperatively. Development of purulent spondylitis because of local infection and postoperative sepsis is a problem. ${ }^{21-27)}$ One patient with an infection died from sepsis and purulent spondylitis. AEF was suspected from the time of initial EVAR, but it was observed after the EVAR because there was no sign of infection. We believe that this delay in treatment caused purulent spondylitis and resulted in sepsis. In cases of infection, if the infection is uncontrollable, early operation should be considered.

\section{Conclusion}

Late OC after an EVAR should be considered as a valuable option, especially in cases of repeated failed catheter interventions. In endoleak cases, using an infrarenal aortic clamp including the endograft is safe, but in migration cases with suprarenal stents, there is a risk of aortic injury and attention is necessary. Infection cases require immediate OC because of complications due to sepsis and spread of local infection. 


\section{Disclosure Statement}

None of the authors have any conflict of interest.

\section{Additional Note}

A summary of this report was presented at the 59th Annual Meeting of Japanese College of Angiology on October 25, 2018, in Hiroshima, and received a recommendation by the chairperson.

\section{Authors Contributions}

Study conception and analysis: YN

Data collection: $\mathrm{YN}, \mathrm{KN}, \mathrm{RK}$

Writing: YN

Critical review and revision: all authors

Final approval of the article: all authors

Accountability for all aspects of the work: all authors

\section{References}

1) De Bruin JL, Baas AF, Buth J, et al. Long-term outcome of open or endovascular repair of abdominal aortic aneurysm. N Engl J Med 2010; 362: 1881-9.

2) Sampram ESK, Karafa MT, Mascha EJ, et al. Nature, frequency, and predictors of secondary procedures after endovascular repair of abdominal aortic aneurysm. J Vasc Surg 2003; 37: 930-7.

3) Hobo R, Buth J. Secondary interventions following endovascular abdominal aortic aneurysm repair using current endografts. A EUROSTAR report. J Vasc Surg 2006; 43: 896-902. e1.

4) Brinster CJ, Fairman RM, Woo EY, et al. Late open conversion and explantation of abdominal aortic stent grafts. J Vasc Surg 2011; 54: 42-6.

5) Chaar CIO, Eid R, Park T, et al. Delayed open conversions after endovascular abdominal aortic aneurysm repair. J Vasc Surg 2012; 55: 1562-9.e1.

6) Gambardella I, Blair PH, McKinley A, et al. Successful delayed secondary open conversion after endovascular repair using partial explantation technique: a single-center experience. Ann Vasc Surg 2010; 24: 646-54.

7) Bonvini S, Wassermann V, Menegolo M, et al. Surgical infrarenal "neo-neck" technique during elective conversion after EVAR with suprarenal fixation. Eur J Vasc Endovasc Surg 2015; 50: 175-80.

8) EVAR trial participants. Endovascular aneurysm repair versus open repair in patients with abdominal aortic aneurysm (EVAR trial 1): randomized controlled trial. Lancet 2005; 365: 2179-86.

9) Patel R, Sweeting MJ, Powell JT, et al. Endovascular versus open repair of abdominal aortic aneurysm in 15-years' follow-up of the UK endovascular aneurysm repair trial 1 (EVAR trial 1): a randomised controlled trial. Lancet 2016; 388: 2366-74.

10) Blankensteijn JD, de Jong SE, Prinssen M, et al. Two-year outcomes after conventional or endovascular repair of abdominal aortic aneurysms. N Engl J Med 2005; 352: 2398405.

11) Patel SR, Allen C, Grima MJ, et al. A systematic review of predictors of reintervention after EVAR: guidance for riskstratified surveillance. Vasc Endovascular Surg 2017; 51: 417-28.

12) May J, White GH, Yu W, et al. Conversion from endoluminal to open repair of abdominal aortic aneurysm: a hazardous procedure. Eur J Vasc Endovasc Surg 1997; 14: 4-11.

13) Kouvelos G, Koutsoumpelis A, Lazaris A, et al. Late open conversion after endovascular abdominal aortic aneurysm repair. J Vasc Surg 2015; 61: 1350-6.

14) Matsagkas M, Kouvelos GN, Peroulis $M$. Safe and fast proximal aortic control using an aortic balloon through direct graft puncture for the explantation of an abdominal endograft with suprarenal fixation. Interact Cardiovasc Thorac Surg 2014; 18: 519-21.

15) Klonaris C, Lioudaki S, Katsargyris A, et al. Late open conversion after failed endovascular aortic aneurysm repair. J Vasc Surg 2014; 59: 291-7.

16) Lipsitz EC, Ohki T, Veith FJ, et al. Delayed open conversion following endovascular aortoiliac aneurysm repair: partial (or complete) endograft preservation as a useful adjunct. J Vasc Surg 2003; 38: 1191-7.

17) Hinchliffe RJ, Singh-Ranger R, Whitaker SC, et al. Type II endoleak: transperitoneal sacotomy and ligation of side branch endoleaks responsible for aneurysm sac expansion. J Endovasc Ther 2002; 9: 539-42.

18) Mangialardi N, Ronchey S, Orrico M, et al. Surgical conversion with graft salvage as a definitive treatment for persistent type II endoleak causing sac enlargement. J Vasc Surg 2015; 62: 1437-41.

19) Moulakakis KG, Klonaris C, Kakisis J, et al. Treatment of type II endoleak and aneurysm expansion after EVAR. Ann Vasc Surg 2017; 39: 56-66.

20) Maitrias P, Belhomme D, Molin V, et al. Obliterative endoaneurysmorrhaphy with stent graft preservation for treatment of type II progressive endoleak. Eur J Vasc Endovasc Surg 2016; 51: 38-42.

21) Fiorani P, Speziale F, Calisti A, et al. Endovascular graft infection: preliminary results of an international enquiry. $\mathrm{J}$ Endovasc Ther 2003; 10: 919-27.

22) Vogel TR, Symons R, Flum DR. The incidence and factors associated with graft infection after aortic aneurysm repair. J Vasc Surg 2008; 47: 264-9.

23) Chaufour X, Gaudric J, Goueffic Y, et al. A multicenter experience with infected abdominal aortic endograft explantation. J Vasc Surg 2017; 65: 372-80.

24) Ducasse E, Calisti A, Speziale F, et al. Aortoiliac stent graft infection: current problems and management. Ann Vasc Surg 2004; 18: 521-6.

25) Lynns OTA, Baguneid M, Barwick TD, et al. Diagnosis of aortic graft infection: a case definition by the management of aortic graft infection collaboration (MAGIC). Eur J Vasc Endovasc Surg 2016; 52: 758-63.

26) Argyriou C, Georgiadis GS, Lazarides MK, et al. Endograft infection after endovascular abdominal aortic aneurysm repair: a systematic review and meta-analysis. J Endovasc Ther 2017; 24: 688-97. 
27) Li HL, Chan YC, Cheng SW. Current evidence on management of aortic stent-graft infection: a systematic review and meta-analysis. Ann Vasc Surg 2018; 51: 306-13.

28) Kelso RL, Lyden SP, Butler B, et al. Late conversion of aortic stent grafts. J Vasc Surg 2009; 49: 589-95.
29) Usatii A, Payne W, Santilli S. Removal of an infected aortic endograft and open aortic reconstruction: technical remarks. Ann Vasc Surg 2013; 27: 679-83.

30) Youn JK, Kim SM, Han A, et al. Surgical treatment of infected aortoiliac aneurysm. Vasc Spec Int 2015; 31: 41-6. 